\title{
SULL'INFLUENZA SOLARE NELLE ESPERIENZE DI GIROINTERAZIONE
}

\author{
G. RighinI - G. GodoLI
}

Le esperienze di girointerazione che l'Istituto di Fisica tecnica dell'Università di Napoli ha organizzate, sotto la direzione di M. Cutolo, dal 1946 hanno mostrato che il fenomeno si verifica soltanto in modo saltuario.

Tale fatto aveva indotto i ricercatori a pensare che le perturbazioni cromosferiche, connesse con l'attività generale e sporadica del вole, fossero responsabili di questo; è proprio per indagare una even-

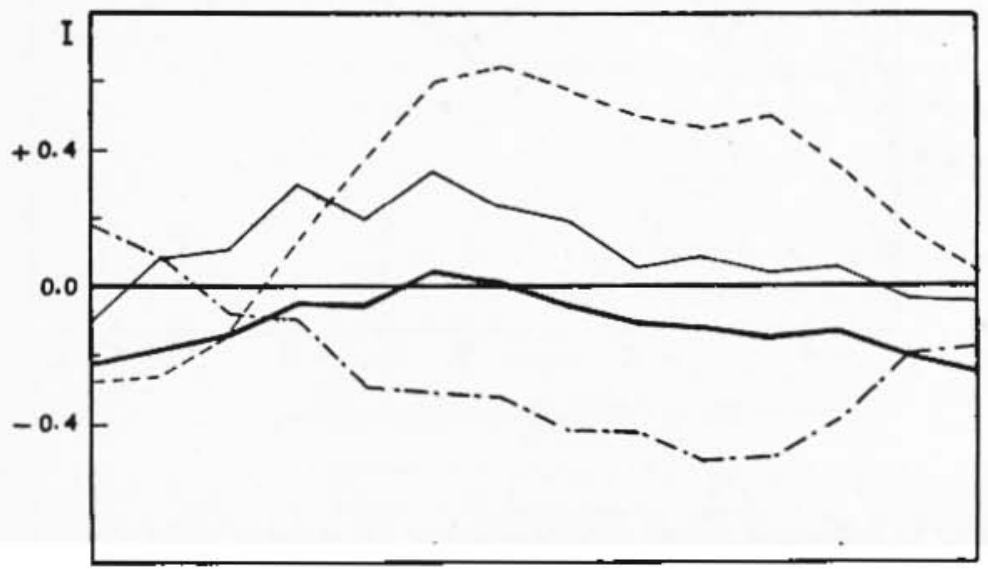

tuale influenza del sole sulle esperienze di girointerazione che abbiamo intrapreso questo lavoro su richiesta del centro studi per la radiopropagazione dell'Università di Napoli.

Il problema, oltre al suo interesse speculativo, rivestiva una certa importanza pratica in vista di ulteriori esperienze: data infatti la loro complessità, qualora fosse stata messa in luce una qualche in- 
fluenza solare diretta od indiretta del fenomeno, ci si sarebbe astenuti dall'eseguire ricerche nei giorni di maggiore attività solare.

Ci siamo limitati a prendere in considerazione le esperienze dello campagne del 1948-1950 eseguite nei giorni contenuti nella tabella I: tali esperienze erano effettuate con omogeneità di metodo e risulıavano le più attendibili. Nella tabella sono sottolineati i grorni in cui l'interazione non ha avuto luogo.

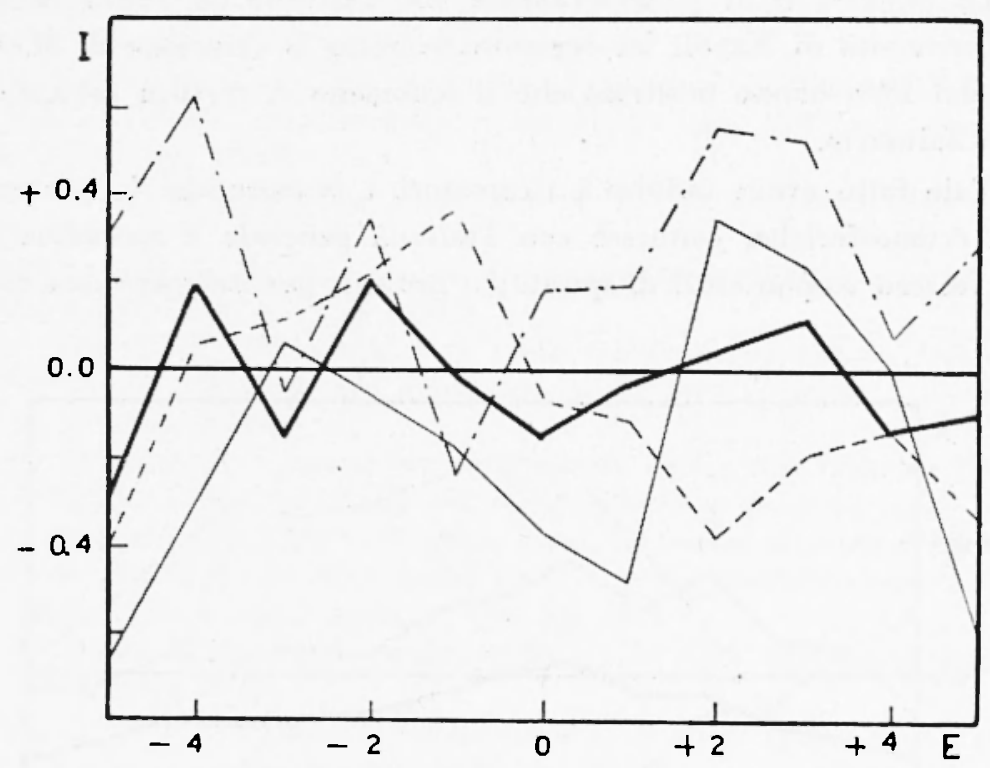

Fig. 2 - Protuberanze al meridiano.

Per le influenze solari dirette, dovute all'attività generale del sole, è stato studiato il comportamento delle macchie e delle protuberanze al meridiano centrale del sole mentre per le influenze dirette, dovute ai fenomeni sporadici, abbiamo preso in considerazione $i$ brillamenti solari. L'influenza indiretta del sole è stata studiata prendendo in considerazione gli indici triorari $K$, che caratterizzano lattivita magnetica generale, le perturbazioni ad inizio brusco $S c$, che sono una conseguenza della radiazione corpuscolare di origine solare, ed infine i "crochet " magnetici o perturbazioni brusche di breve durata che sono direttamente connessi ai bruschi aumenti di radiazione ultra- 


\section{Tabella I}

Giorni nei quali furono esezuite esperienze di girointurazions. Sono sottolineati i giorni in cai l'intartzions non ha avuto luogo.

\begin{tabular}{l}
19.18 \\
$\frac{12}{15} . \mathrm{V}$ \\
13 \\
$2: 1$ \\
22 \\
25 \\
$\frac{27}{29}$ \\
$\therefore 1$ \\
\hline 1 \\
3 \\
5 \\
4
\end{tabular}

1949

$18 . . . \mathrm{VI}$

19

21

23

\section{$\overline{28} \ldots, 11$}

$-\overline{2} \ldots$ VIII

3

6

8

9

13

15

TABEILA II

Grandeza assunte per i vari fenomeni considerati
3950

$$
10 \ldots V
$$$$
\overline{20}
$$

24

30

$$
\begin{aligned}
& \frac{1}{4} \ldots \text { VI } \\
& \frac{10}{\frac{13}{17}} \\
& \frac{17}{23} \\
& 1 \ldots \text { VII }
\end{aligned}
$$$$
\text { 3... Vill }
$$

Fenomeno

Macchie

Protulieranze al meridiano

Brillamenti

Indici $K$

Sc

Crocliet

Frequenza critica dello strato $F$.

Frequenza minima riflessa dallo strato $D$ Assorlimento dello strato $D$

\section{Grandezza}

Numero di Wolf $(1)$.

Attivita delle protuberanze al bordo (nei griorni opportuni| espressa in unità di protuberanza (2).

Numero griornaliero (3).

Somma degli 8 valori riornalieri (4).

Numero gionaliero (5).

Somma griornaliera dei prodotti $a \cdot b . c$ (6) dove $t=$ numero di osservatori registranti il fenomeno

b. durata' $15^{\text {t" }}$

$c=1$ se il fenomeno non è stato con. fermato da osservazioni solari od ionosferiche

c. -2 se il fenomeno $\dot{e}$ stato confermato.

$f_{1} F$. alle ore $12(7 \mathrm{r}$

$f D$ alle ore $12(8)$.

$A D$ alle ore $12(9)$. 


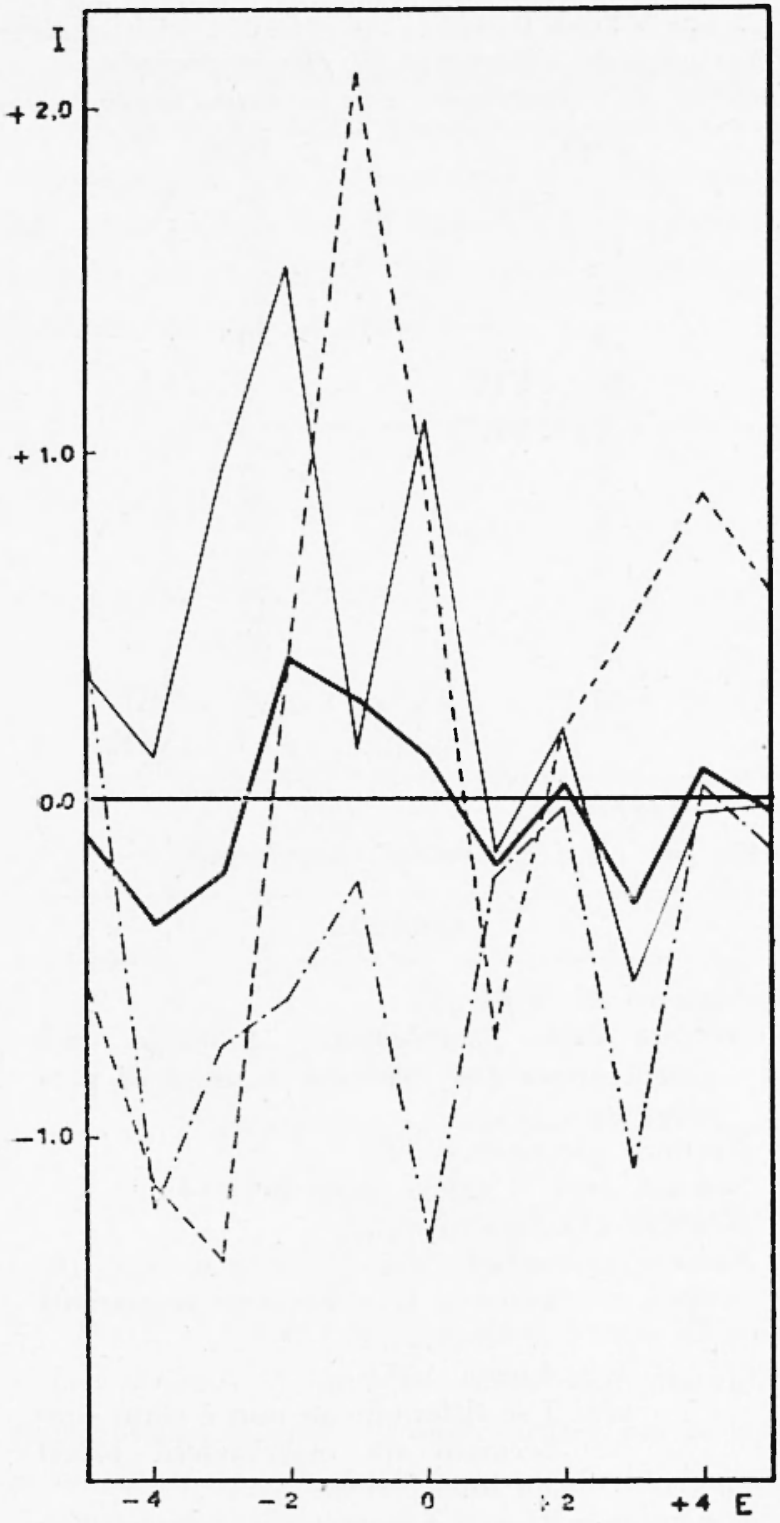

Fig. 3 - Brillamenti. violetta solare. Questi ultimi sono strettamente correlati con i brillamenti solari. Per lo stato della ionosfera abbiamo preso in considerazione la frequenza critica dello strato $F_{2}$ che indicheremo con $f_{0} F_{2}$, quella minima riflessa dallo strato $D$ che indicheremo con $f D$ ed infine l'assorbimento dello strato $D$ che indicheremo con $A D$.

Per ognuno dei fenomeni considerati è stata scelta una grandezza che ne sintetizzasse l'attività giornaliera; le grandezze assunte sono elencate nella tabella II.

Indicando generalmente con $m$ la grandezza relativa ad un certo fenomeno e con $m_{s}$ il valore che tale grandezza assume in un giorno in cui si osserva il fenomeno della giroin. terazione e con $m^{\mathrm{n}}$ il

valore che essa assume in un giorno in cui non si osserva il fenomeno, abbiamo calcolato le medie seguenti: 


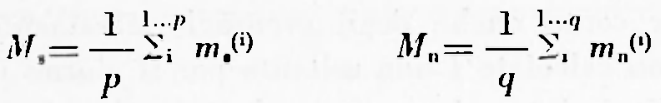

$$
\begin{aligned}
& M=\frac{1}{1+q} \stackrel{1 . q+q}{\because} \quad \text { (i) }
\end{aligned}
$$

che ci danno rispettivamente il valor medio di $m$ per i $p$ gioni di osservazione positiva (si); il valor medio di $m$ per i $q$ giorni di osser-

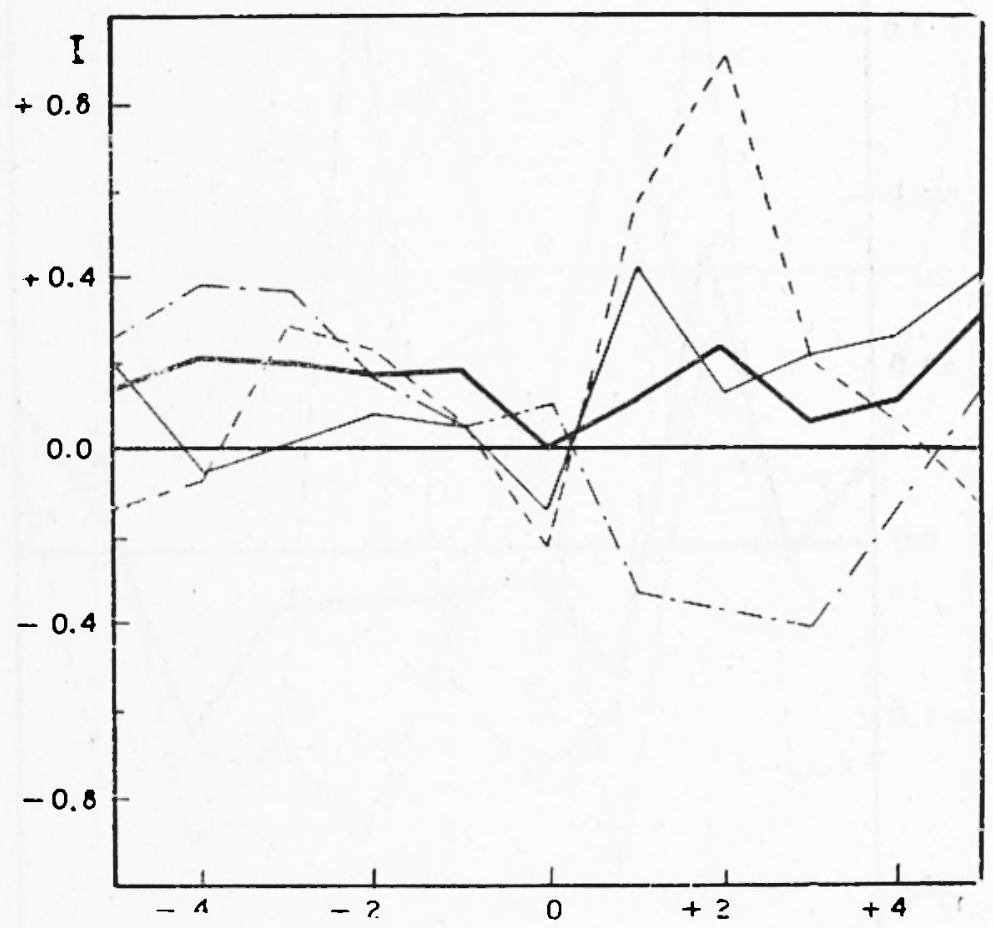

Fig. 1 - Indici K.

vazione negativa (no); ed infine il valore medio di $m$ per tutti i $p+q$ giorni di osservazione.

L'indice statistico $I$ che si deduce dalla

$$
I=\frac{M_{\mathrm{n}}-M_{\text {. }}}{M}
$$

ci dà l'influenza del fenomeno considerato sulla girointerazione. Esso evidentemente dovrebbe essere piccolo o nullo se il fenomeno non influisse sulla girointerazione; positivo se il fenomeno agisse come inibitore e negativo nel caso contrario. 
Per tener eonto anche degli eventuali sfasamenti fra causa ed effetto abbiamo calcolato $I$ non soltanto per il giorno in cui si è fatta l'esperienza di girointerazione ma anche per alcuni giorni precedenti e seguenti. Abbiamo cioè applicata a questa indagine il metodo delle

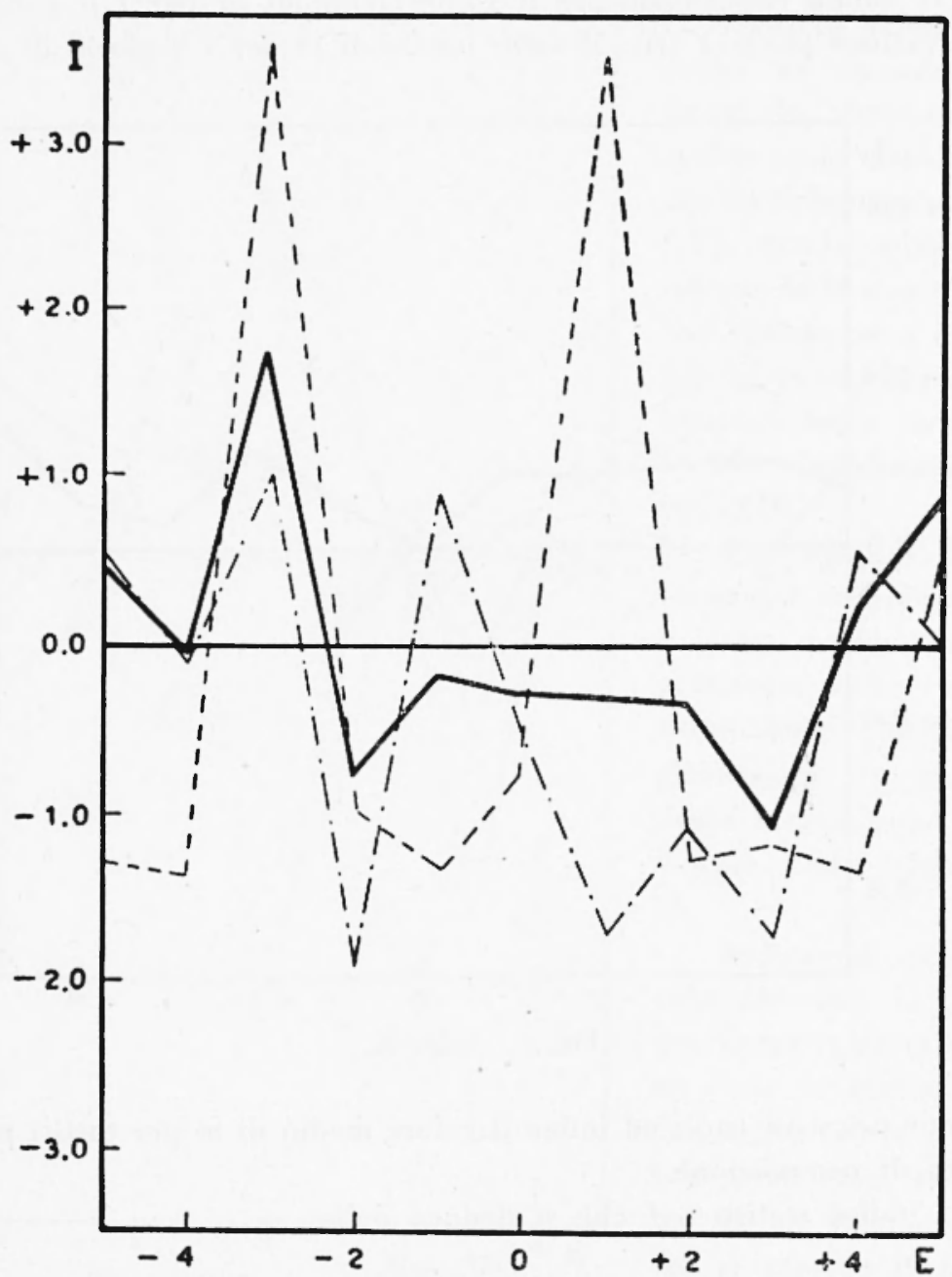

Fig. 5 - Sc.

epoche sovrapposte rappresentando $I$ in funzione dell'epoca $E$. Per il giorno dell'osservazione si avrà $E=0$; per il giorno precedente $E=-1$, per quello seguente $E=+1$ e così via. 


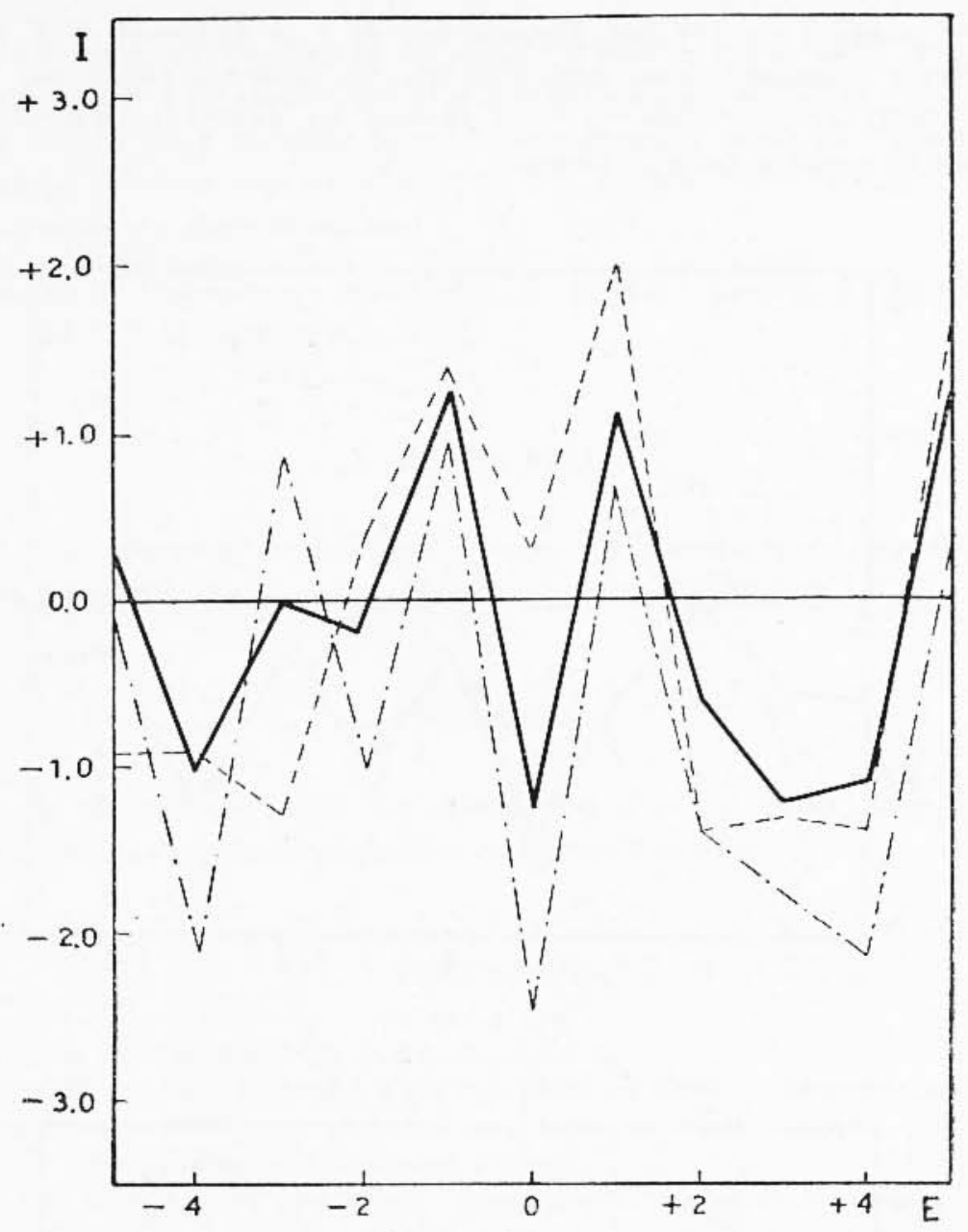


I grafici $1 \div 7$ danno l'andamento di $I$ in funzione di $E$ per i diversi fenomeni. Sono stati tracciati tre grafici separati per gli anni $1948^{(-)}-49^{(\ldots . .)}-50^{(---)}$ed inolire un quarto grafico estendendo le medie a tutto il triennio (-).

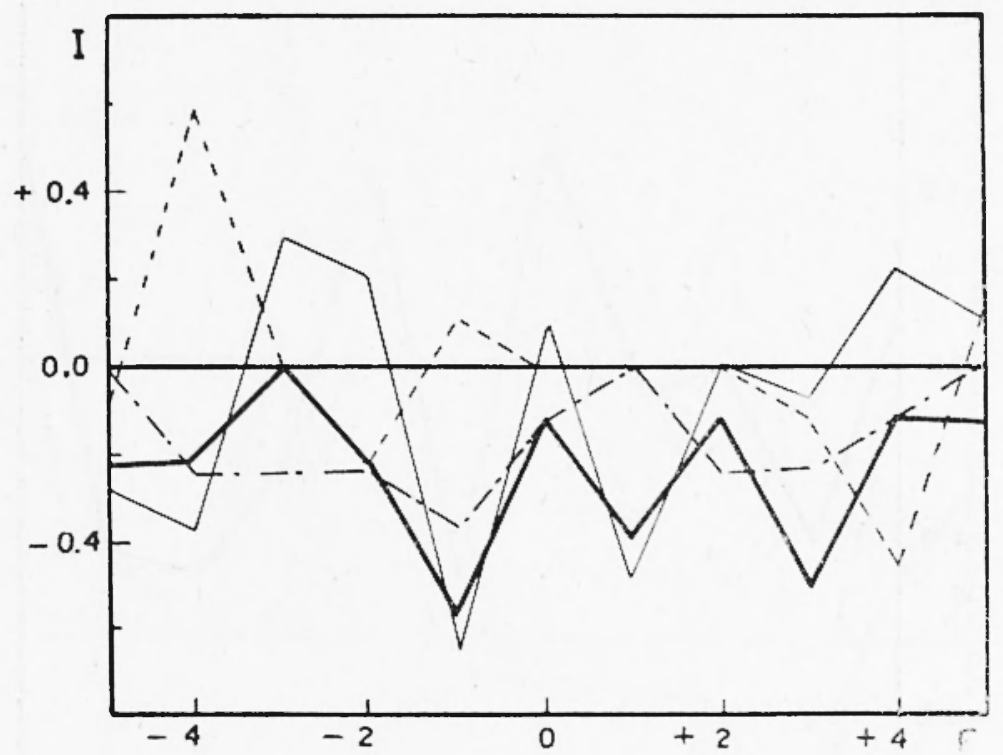

Fig. 8 - fD.

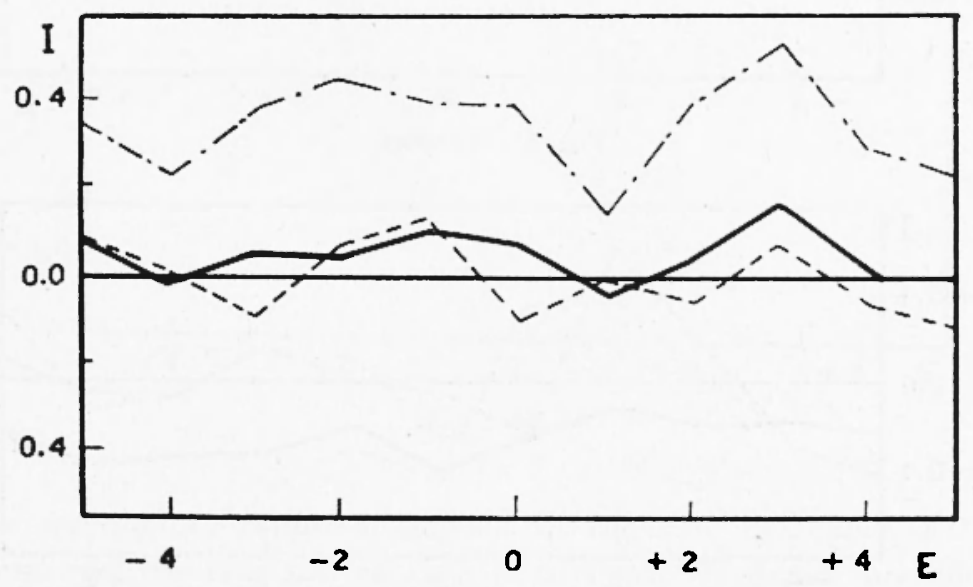

Fig. 9 - AD. 
Il materiale ha ancora un valore statistico molto basso per poterne trarre delle conclusioni sicure e dettagliate; possiamo però asserire che in linea generale le varie perturbazioni solari ed i fenomeni geofisici connessi, non sembrano responsabili dei risultati negativi delle esperienze di girointerazione.

Firenze - Osservatorio Astrofisico di Arcetri - Centro di Astrofisica del C.N.R. - Febbraio 1952.

\section{RIASSUNTO}

Nella presente nota si mostra come sia estremamente improbabile una influenza solare nelle esperienze di girointerazione.

\section{SUMMARY}

In the present paper we shoun that it is extremely improbable that there is any solar influence on gyro-interaction.

\section{BIBLIOGR AFIA}

(1) e (3) Quarterly Bulletin on solar activity.

(2) Osservazioni di Arcetri e Catania ridotte ad Arcetri e conservate negli archivi dell'osservatorio.

(4) (5) (6) Journal of Geophysical Research.

(7) (8) (9) S.P.I.M. Observations Ionospheriques. Station de Fribourg. 